\title{
Idées fondamentales quant à une «loi sur la prévention» 1
}

\section{Martha Ribi ${ }^{2}$}

Groupe de travail «Législation». Présidente: Mme Martha Ribi, conseillère nationale, Zurich. Experts: Dr U. Frey, Berne; Dr J. Martin, Lausanne.

Le groupe de travail «Législation» entendit tout d'abord deux exposés introductifs, l'un sur les actions législatives envisageables aux trois niveaux de la prévention primaire, secondaire et tertiaire $(\mathrm{Dr}$ J. Martin), l'autre sur les travaux préparatoires à propos d'une loi fédérale sur la prévention ( $\mathrm{Dr}$ $U$. Frey). II discuta ensuite la question fondamentale de savoir si un tel cadre législatif est nécessaire et, le cas échéant, à quels principes il devrait obéir. Après discussion, les conclusions du groupe ont été les suivantes:

1. Une loi fédérale sur la prévention devrait être une loi-cadre, qui ne devrait pas restreindre la compétence des cantons, à qui est pour l'essentiel dévolue la responsabilité dans le domaine sanitaire.

2. Cela ne devrait être ni une loi «de subvention», ni une loi «de police».

3. Le groupe considère qu'une telle loi aurait entre autres et de façon utile une valeur de symbole (d'un engagement de la collectivité en faveur de la prévention).

4. La loi devrait mettre l'accent sur ce qui est réalisable, sinon il ne sera pas possible de la faire adopter sur le plan politique.

Sur la base de ces considérations et à la suite d'un échange de vues approfondi entre les membres du groupe, les idées suivantes furent formulées:

\section{But}

Protection et promotion de la santé par l'intermédiaire de mesures éducatives et préventives. La coordination avec les lois fédérales pertinentes déjà en vigueur reste réservée.

\section{Domaine d'application}

Risques pour la santé (facteurs de risque), qui peuvent causer (ou favoriser) des maladies, des infirmités ou des accidents dont la gravité, la fréquence, les séquelles invalidantes et les conséquences financières pour l'individu, sa famille et/ou la société sont particulièrement marquées, et qui peuvent être évitées ou favorablement influencées par des mesures préventives.

\footnotetext{
'Rapport du groupe de travail «Législation» lors du congrès Forum Davos 78: Limites de la médecine III, La prévention et ses possibilités.

2 Lic oec. publ., Conseillère nationale, Kilchbergstrasse 45, 8038 Zürich.
}

En particulier, ce domaine d'application s'étend à:

- Abus d'alcool, de tabac, de médicaments et de stupéfiants

- Autres risques pour la santé comme l'alimentation inadéquate et le manque d'exercice

- Hygiène dentaire

- Hygiène mentale

\section{Tâches des cantons}

- Promotion de l'éducation pour la santé Information adéquate des groupes de population particulièrement «à risque»

Amélioration des conditions du milieu dans une optique d'écologie humaine

- Promotion de la formation de base et de la formation continue (perfectionnement) du personnel technique concerné.

La collaboration intercantonale doit être recherchée.

\section{Tâches de la Confédération}

- La Confédération apporte aux cantons et aux organisations privées un support par des prestations de services (par exemple, statistiques, documentation, information).

- Elle s'attache à la promotion et à la coordination de l'activité des cantons et des organisations privées sur le plan national.

- Elle peut mettre en œuvre un organe de coordination consultatif, auquel cas il convient de tenir compte des commissions existantes.

- Elle peut exécuter des mesures préventives au niveau de l'ensemble du pays, en collaboration avec les cantons et les organisations privées.

- Elle encourage la recherche scientifique dans les domaines de l'éducation pour la santé et d'autres mesures préventives.

\section{Organisations privées}

La Confédération et les cantons peuvent d'un commun accord déléguer certaines tâches ou attributions à des organisations privées.

\section{Financement}

Il est nécessaire d'inclure au budget des moyens financiers adéquats pour l'exécution des tâches confiées à la Confédération. Il y a lieu d'étudier quelle est l'importance des sommes requises et si de nouvelles sources de financement peuvent être trouvées.

Le principe de causalité (Verursacherprinzip) doit être pris en compte de façon approrpiée. 